\title{
Syllable weight and duration: A rhyme/intervals comparison
}

\author{
Anya Lunden*
}

\begin{abstract}
Steriade (2012) proposed intervals as a more appropriate syllable weight domain than rhymes. This study explores how interval weight cashes out as duration across word positions and compares this to a rhyme-based account. The data reported on in Lunden (2013), from native speakers of Norwegian (a language in which (C)VC syllables are heavy only non-finally) is reanalyzed with intervals. Lunden found that syllable rhymes in all three positions, if taken as a percentage of the average $\mathrm{V}$ rhyme in that word position, fell into a coherent pattern for weight. It is shown that interval durations allow for a similar, albeit less robust, pattern. The data from Lunden's (2013) perception experiment that tested the correlation between increased vowel duration and listeners' classification of syllable weight is also recast with interval durations, and the importance of the proportional increase over the raw increase, originally found for the rhyme data, is found to hold for the interval data. Thus, taking intervals as the weight domain is shown to result in reasonable durational relations between interval weights, although interval durations show less separation between some light and heavy units than the rhyme durations do.
\end{abstract}

Keywords. syllable weight; duration; rhymes; intervals

1. Introduction. Steriade (2012) has proposed that weight is best calculated over intervals, which span from the beginning of a vowel through all following consonants up to the next vowel. This differs from the traditional view that syllable rhymes are the domain for syllable weight (McCarthy 1979). This means that rhymes and intervals will include the same segments under two conditions: (i) word-finally and (ii) hiatus. Otherwise, intervals are always larger than the rhyme equivalent of the same weight domain. This paper examines the consequences of the first of these (wordfinal weight domain equality), and compares the durations of weight domains in non-final and final positions under a rhyme account and an interval account. The difference in non-final weight domains as well as the lack of difference in the final weight domains between rhyme theory and interval theory is illustrated in (1).

(1) Rhymes vs. Intervals

Rhymes Intervals
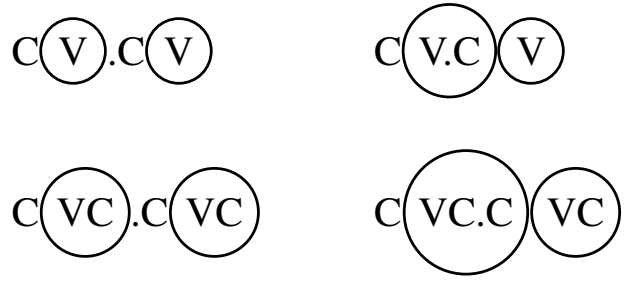

*I thank Robin de Azagra for her assistance with measuring the data and her interest in discussing the work. I thank the audience at LSA 2017 for their comments and feedback. Any mistakes are my own. Author: Anya Lunden, College of William \& Mary (lunden@wm.edu). 
Two types of evidence have been offered in favor of intervals over syllable rhymes as the relevant domain for weight. The first is of the sort presented in Ryan (2014) and Garcia (2017) where corpora are examined and a correlation has been found between the number of segments in an interval and its weight behavior. The second is of the sort discussed in Hirsch (2014) where a production experiment shows the stress-drawing effect of larger intervals. I am not aware of any durational evidence regarding intervals, or any previous comparison of rhyme and interval durations.

Weight is generally accepted to cash out phonetically as duration. This expectation is particularly clear when looking at the encoding of geminates in moraic theory. A single consonant that has an underlying unit of weight (a mora), is realized as a geminate when syllable structure is such that the consonant occurs before a vowel, and so must serve as both the coda of one syllable and the onset of the following syllable, and thus be realized as a long consonant. More generally, there have been various phonetic studies that have shown direct links between syllable weight and duration, including Broselow et al. (1997), Goedemans (1998), Gordon (2002), and Lunden (2011, 2013).

Here, the sound files from the production experiment with native Norwegian speakers reported in Lunden (2013) are used to compare interval and rhyme measurements. This data is wellsuited to a comparison of the two theories, because nonce words of three syllables, all containing the vowel [a] were used, meaning that effects on syllable/interval duration due to syllable/interval size can be examined without regard to inherently differing vowel durations (Lehiste 1970).

1.1 Final Syllable Weight. Many languages have a different criterion for word-final syllable weight. In such languages, a tautosyllabic short vowel and coda consonant will count as heavy anywhere but word-finally. Phonological theory has proposed two different ways to make final syllables have the same weight criterion as non-final syllables: final consonant extrametricality and interval theory.

Final consonant extrametricality treats a word-final consonant as not contributing to the weight of the last syllable. Thus, a word-final CVC syllable would, for the proposes of any weightsensitive phenomena, be CV.C, and therefore light, like any other CV syllable. This approach also offered an explanation for why many languages permit (C)VXC syllables only in word-final position. Since the final consonant is not part of the last syllable, the syllable itself is (C) VX, a normal heavy syllable. In this way final consonant extrametricality made the segmental weight criterion within a language consistent between all syllables (i.e. (C)VX is heavy).

Interval theory takes the segmental span relevant for weight to be vowel-to-vowel; that is, from the vowel of a syllable through all consonants up to but not included the next syllable's vowel. This again has the effect of leveling the segmental criterion for weight, but instead of taking away a segment from the final syllable, it (in the most straightforward case) adds a segment to all nonfinal syllables. Thus, a $\mathrm{C} * \mathrm{~V} . \mathrm{C} * \mathrm{VC}$ word has two light intervals (marked with ' $*$ '), both of which are VC. Heavy intervals are then VXC in all positions, as in $\mathrm{C} * \mathrm{VC} . \mathrm{C} * \mathrm{VCC}$ which consists of two heavy intervals. 
(2) Segment-based accounts of a consistent weight condition across positions

\begin{tabular}{l|ll} 
& heavy & adjustment \\
\hline rhymes & VX & word-final C discounted: $\mathrm{VX}<\mathrm{C}>\#$ \\
intervals & VX.C & [none]
\end{tabular}

Lunden (2013) proposed a phonetic criterion for syllable weight based on data from Norwegian, which relies on the inherent additional phonetic length that is present word-finally. Finallengthening affects the right-edge of prosodic constituents, including the prosodic word (Oller 1973). Norwegian is a language in which heavy rhymes must be VXC in word-final position, but elsewhere treats VX as heavy. The language also has the requirement that the syllable bearing primary stress must be heavy, thus making it a helpful language to look at for the interplay between duration and weight.

Rather than a weight domain specification that produces segmental equivalence, as the two in (2) do, Lunden shows that the increase in duration of a heavy rhyme over a short vowel in the same position of the word regularly meets a proportional increase threshold. In Norwegian, she found that heavy rhymes typically have a proportional increase in duration of at least $50 \%$ of a $\mathrm{V}$ rhyme in the same position of the word. Crucially, while a VC rhyme was found to meet this threshold in non-final positions, with an average of a $64 \%$ increase over a non-final $\mathrm{V}$ rhyme, a word-final VC rhyme did not. In word-final position, a VC rhyme had, on average, a 36\% increase over a final V rhyme, while a VXC rhyme had an average increase of at least $70 \%$. This is because word-final rhymes are longer to begin with, and so require additional raw duration to reach the same proportional increase that is found with fewer segments in non-final positions.

Lunden proposes that it is the proportional increase, rather than the raw increase, in duration that is relevant for syllable weight. This follows from the fact that human perception works in this way- we need ever-bigger raw increases to maintain linearly-increasing perception, which is why many perceptual scales (e.g. decibels, octaves) are logarithmic.

Rather than adjusting what segments count in the weight domain, the proportional increase approach crucially relies on the non-final/word-final difference in number of segments in a heavy syllable, as the phonetic criterion of a threshold proportional increase can then be applied consistently across all positions.

(3) Weight criteria proposals

\begin{tabular}{llll} 
& & criterion & note \\
\hline segmental & i. & rhyme is at least VX & discount a word-final consonant \\
& ii. & interval is at least VXC & \\
phonetic & iii. & $\begin{array}{l}\text { rhyme has at least the minimum } \\
\text { proportional increase over } \\
\end{array}$ & \\
& & same-position V rhyme
\end{tabular}

Both the interval and proportional increase accounts have the advantage of not needing to single out the word-final position in any way. ${ }^{1}$

\footnotetext{
${ }^{1}$ The final consonant extrametricality account, while needing a special stipulation about the right edge, can be seen
} 
1.2 Norwegian Data, Remeasured. The Norwegian data used is from production and perception studies originally reported in Lunden (2013). For the production study, fourteen native Norwegian speakers read nonce words of the basic form katapa in a carrier phrase. Coda consonants were added in different versions, e.g. kantapa, kantappa, etc. Long vowels were not directly marked, as a stressed open syllable will be pronounced with a long vowel. One syllable per word was capitalized to indicate target stress placement, but resulting data was coded with the actuallypronounced stress.

The original dataset was narrowed for the present comparison. Stimuli with geminates as well as those with diphthongs were excluded because the focus here is not to categorize all syllable shapes by weight, but to show the relative durations of key syllable types. Those stimuli that included a stop coda were put aside because these were common word-finally but rare non-finally. Thus the data in the current dataset includes only open syllables and those with a nasal coda. The complex word final coda [ $\mathrm{nk}]$ was also included so that final VCC rhymes/intervals could be examined. In the interval data, non-final open syllables of course include the stop of the following syllable, so all non-final light intervals are VT (where ' $\mathrm{T}$ ' represents a voiceless stop). (Wordfinally, both V and VT are light.)

The original delineation of the data included both the voiceless portions of vowels and the release of stops. While some non-final vowels included a devoiced portion, this was mainly an issue for word-final vowels which often had a relatively long trail-off period. Further, word-final stops sometimes had a notably long release. In this remeasurement, only the voiced portion of the vowels and the closure portion of the stops were included. Two example delineations are shown in (4). The higher textgrid tier delineates rhymes, the lower delineates intervals. Capitals indicate the stressed rhyme or interval.

(4) Sample delineations

a.

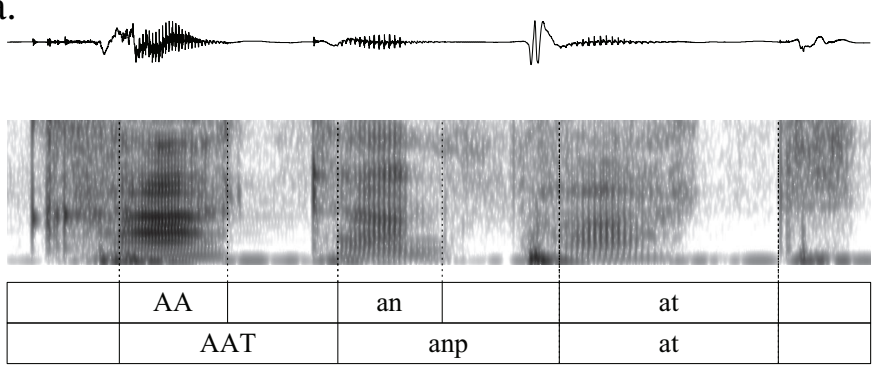

b.

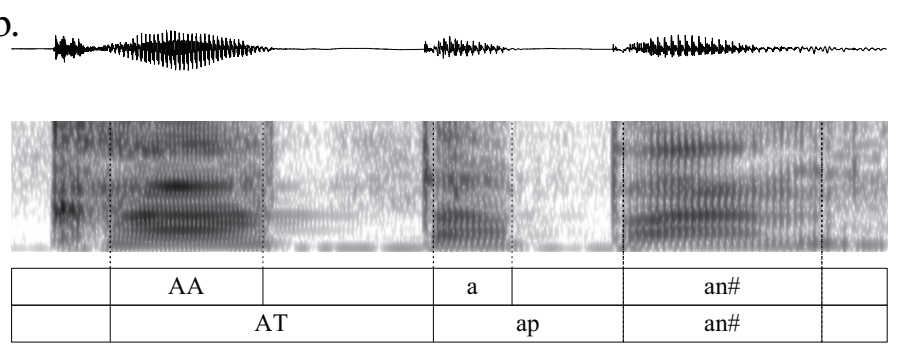

as a type of NONFinALITY (Prince \& Smolensky 1993): a different way of satisfying the pressure to not have a foot (or, in some accounts, a stressed syllable) be word-final than occurs with final syllable extrametricality. 
2. Rhyme and interval comparisons. A very clear link between duration and weight was found using rhymes as the weight domain by Lunden (2013). No published work that I am aware of has examined the link between duration and intervals as weight domains. We will look both at the raw durations as well as the proportional increases, both for the production study outlined in the previous section, and also with regards to a related perception study.

It is a strength of both intervals and proportional increase with rhymes that word-final syllables can be seen to behave the same as non-final weight domains. For proportional increase with rhymes, this is true looking at a phonetic durational comparison, but is not true looking at the segmental content of final and non-final domains (as more segmental content is needed in the final rhyme in order to reach the needed proportional increase). For intervals, the segmental content of both domains is the same. It is unknown whether it is true for the phonetic durational comparisons.

2.1 Duration COMPARISON. The comparisons of raw durations is shown in (5). The shading for the different rhyme/interval shapes is kept equivalent, not equal, between the two. For example, a non-final V rhyme is empty white, as is a non-final VC interval, as these are two proposals for the weight domain of a syllable with a short vowel followed by a syllable with an onset. The durations of the final rhymes and intervals are identical, as the final weight domains are identical between the theories. The effect of final lengthening is evident in the final rhymes. The final intervals, however, are somewhat shorter than the non-final intervals because the non-final intervals include the onset consonant of the following syllable.

\section{Raw Duration}

a. Rhymes

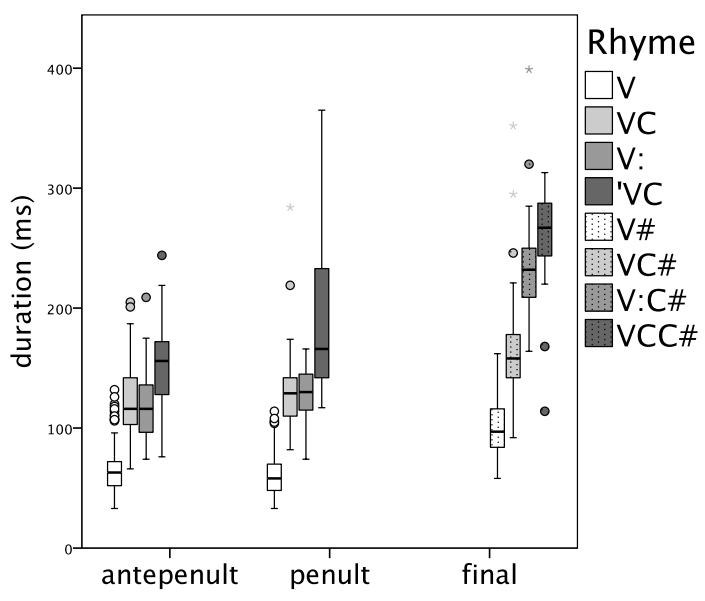

b. Intervals

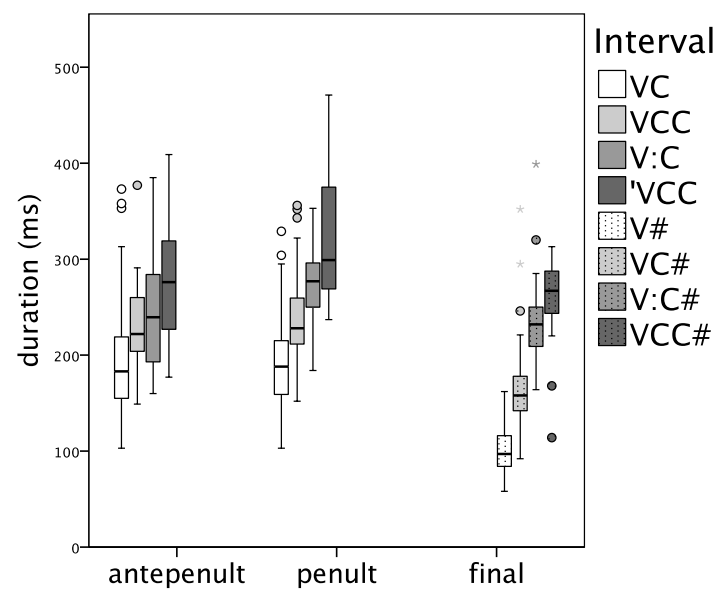

The light weight domains are the shortest ones in non-final positions and the two shortest ones in final position. The rhyme durations, in (5a), do not show any consistent durations by weight across positions, due to the effects of final lengthening on the final syllables. Looking at distributions of the interval data, on the other hand, we see a reasonable separation between the durations of light and heavy syllables. That is, the two light word-final intervals (V and VC) are even shorter, on average, than the light non-final intervals (VC); whereas the two heavy word-final 
intervals (V:C and VCC) have average durations on par with the three heavy non-final intervals. I now turn to evidence for the proportional increase criterion for syllable weight.

2.2 PERCEPTION OF DURATION INCREASES. Human perception is logarithmic, not linear (Weber's Law). Perception is not always perfectly logarithmic; for example, our perception of frequency is more linear at lower levels and more logarithmic at higher levels, as encoded in the Bark scale. Across the senses, however, humans need greater raw increases at higher levels to perceive the same increase amount. The same can be seen to be true of duration.

Lunden (2013) ran a perception study in which 34 native Norwegian speakers listened to katapa, katapat stimuli in which one vowel had been lengthened 20, 40, 60, 80, or 100 percent of its unlengthened duration. The baseline durations were taken from the production study averages: $60 \mathrm{~ms}$. for non-final vowels and $130 \mathrm{~ms}$. for final vowels. Onset stop closures were $65 \mathrm{~ms}$. and word-final stop closures were $90 \mathrm{~ms}$. Listeners were asked to select which syllable was stressed, where the only cue to stress was vowel length. Since a syllable with primary stress must be heavy in Norwegian and no syllables were underlying heavy, only vowel length was available to indicate the position of stress in the word. The graph in (6) shows the correlation between raw increase in duration and correctly-identified syllables (i.e. those with the lengthened vowel) in each of the three positions for the katapat-form words (the kapata-form words were only included to give an explicit baseline for final rhymes). The fit lines for the antepenultimate and penultimate positions are directly on top of each other and cannot be seen as separate lines. The solid line is the overall fit.

(6) Raw increase (Rhymes and Intervals are the same $)^{2}$

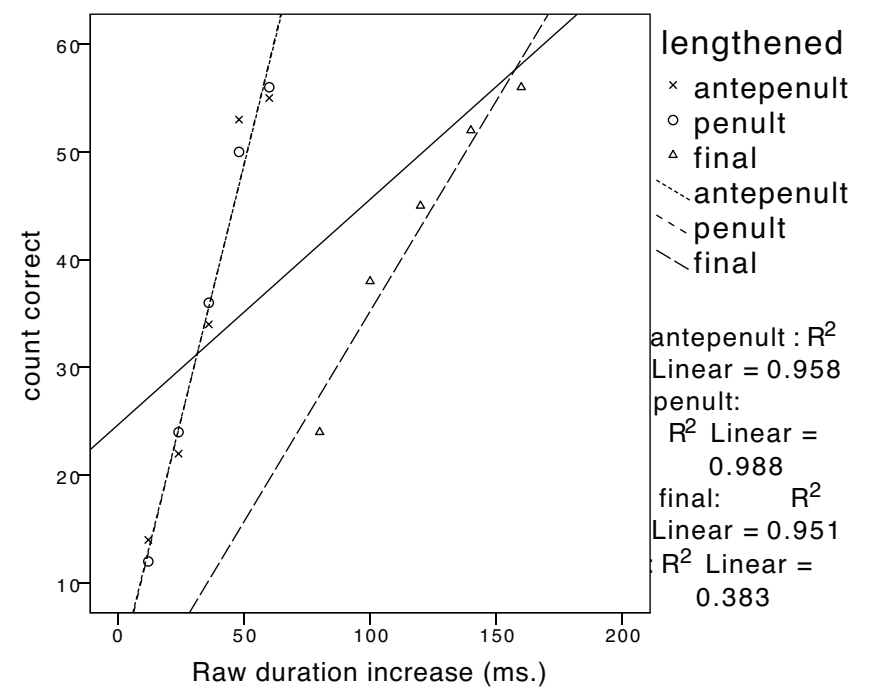

As we would expect, higher increases in duration result in higher numbers of correctlyidentified syllables. However, the scale of the non-final and final positions is very different. We

\footnotetext{
${ }^{2} \mathrm{An}$ almost identical graph was originally published as (25) on page 24 in Lunden (2013) and it is included with permission of Springer, @ Springer Science+Business Media Dordrecht 2013. The present graph varies just slightly in style, and the fit lines have a minor difference as the graph in Lunden (2013) mistakenly graphed 15 ms. raw increase for the first antepenult and penult raw increase instead of $12 \mathrm{~ms}$.
} 
see in the range of 22-24 correct responses with $24 \mathrm{~ms}$. increase in non-final syllables and 24 correct responses with $80 \mathrm{~ms}$. increase in final syllables. If we instead plot the number of correctlyidentified syllables as a function of the proportional increase over the baseline unlengthened vowel duration in each position, a very different picture emerge for rhymes, as shown in (7).

(7) Proportional increase in rhymes ${ }^{3}$

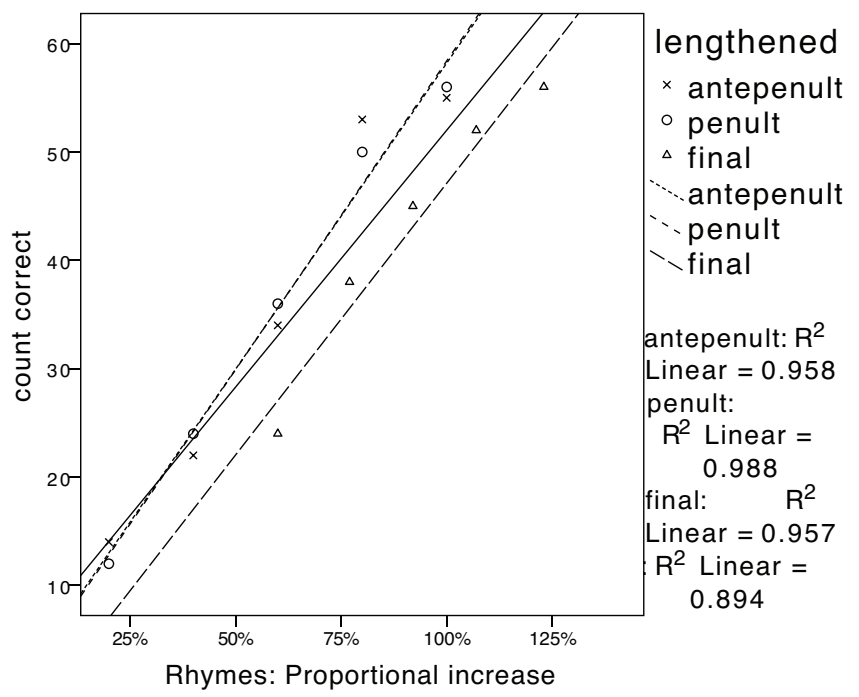

Treating the increases in duration as proportional increases over the baseline duration in the same syllable of word, we see the non-final and final positions behaving the same way. This is consistent with human perception being logarithmic- a greater increase is needed in final position to achieve the same effect as is found with a smaller increase in non-final positions because of the longer "baseline" word-finally, caused by final lengthening.

The graphs in (8) plot the perception data as a proportional increase of each interval. For example, a non-final unlengthened vowel and following consonant together were $125 \mathrm{~ms}$. and so a $12 \mathrm{~ms}$. increase results in an almost $10 \%$ increase (rather than the $20 \%$ increase the additional $12 \mathrm{~ms}$. results in over just the vowel portion alone, as is the case in the rhyme data in (7)). The proportional increases in word-final position were calculated in two ways. For the first, (8a), with the shortest weight domains in each position of the word, just as was done for the rhyme data. This means that a non-final interval will be assessed as an increase over a VC interval, but a final interval will be assessed as an increase over a final $\mathrm{V}$ interval. The first takes the shortest light weight domain in all positions as the baseline. ${ }^{4}$ The second, shown in (8b), takes as a proportional increase over a VC in all positions. Since the strength of interval theory is that VC is a consistent light interval across all positions, it makes sense to think this might be the best choice for a baseline in interval theory. Note again that in both graphs the fit line for the antepenultimate position is

\footnotetext{
${ }^{3}$ This graph has only minor cosmetic differences from the one originally published as (26) on page 25 in Lunden (2013). It is included with permission of Springer, (C) Springer Science+Business Media Dordrecht 2013.

${ }^{4}$ Note that we would have a shorter weight domain non-finally if either the penult or final syllables lacked onset consonants. As having an onset is unmarked, and hiatus is marked, it seems reasonable to take VC as the baseline; however, it is worth noting that with data that included hiatus it would not be the shortest weight domain.
} 
directly on top of that of the penultimate position so the lines cannot be distinguished.

Proportional increase in intervals

a. Shortest weight domain as baseline

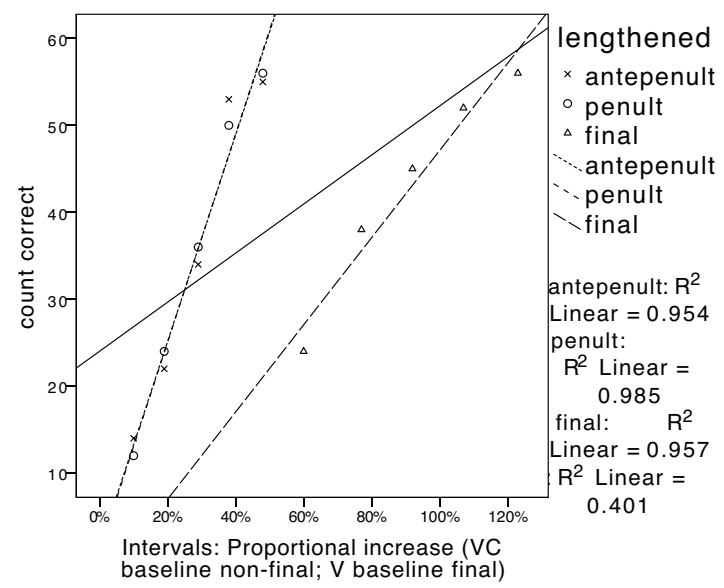

b. $\mathrm{VC}$ in all positions as baseline

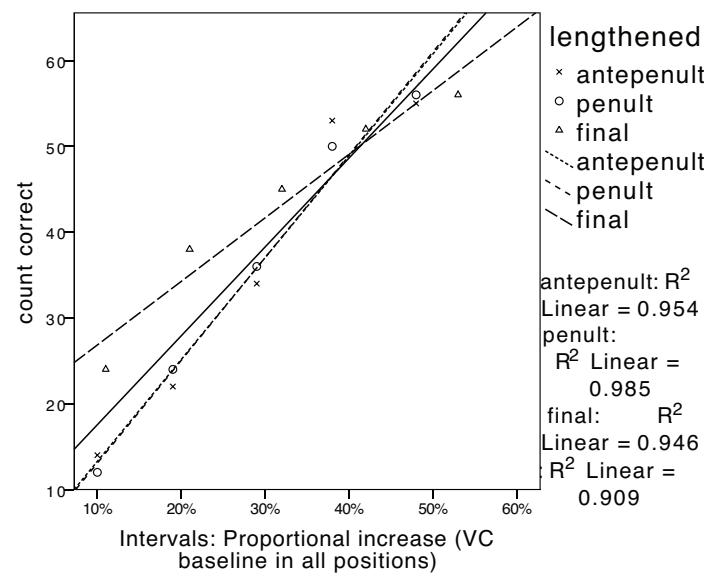

While the graph in (8a), with VC baseline non-finally and a V baseline word-finally, does not show a consistent relationship between proportional increase and correct syllable identification, the graph in (8b), with a consistent VC baseline, does. In fact, it shows the same kind of consistent behavior across positions that (7) does. (The $R^{2}$ of the overall fit lines (of (7) and (8b)) are very similar, although the individual fit lines cross in $(8 \mathrm{~b})$, showing more divergence at lower and higher levels, rather than each position showing the same trajectory as in (7).)

Thus, both the rhyme data and the interval data are consistent with the perceptual evidence for the proportional increase theory of weight, taking $\mathrm{V}$ as the baseline for the rhyme data and VC as the baseline for the interval data.

2.3 PROPORTIONAL INCREASE COMPARISON. We now want to examine the weight domain measurements from the production experiment with respect to their proportional increases over each position's baseline. Following the methodology of Lunden (2013), for the rhyme data, the average $\mathrm{V}$ rhyme for each position (antepenultimate, penultimate, final) was calculated, and then each rhyme duration was divided by the average $\mathrm{V}$ for that position. The unstressed short vowel was used as the baseline since it is the rhyme shape that is light in all positions. The proportional increases for four rhyme shapes are given in each position in (9). The crucial comparison is between V (white) and unstressed VC (lightest gray) rhymes in each position. 
(9) Rhymes as percentage of same-position V

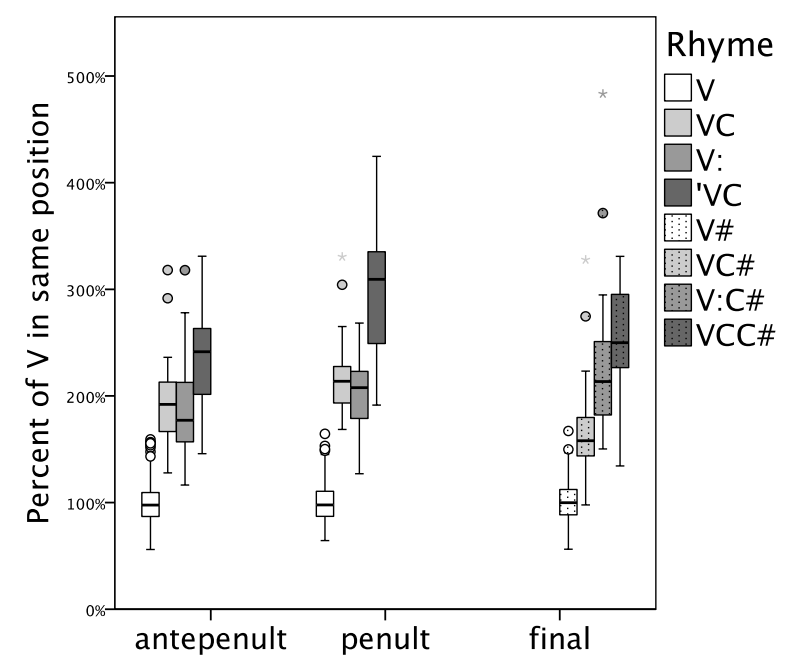

As each rhyme is taken as a percentage of the average $\mathrm{V}$ rhyme in the same position of the word, we no longer see the overall longer durations that were evident when looking at the raw durations of the rhymes in (5a). Comparisons between non-final and final unstressed VC rhymes are critical because this is the one non-final heavy syllable shape that can occur unstressed (as long vowels only occur under primary stress). We see three levels for the average same-position percentage between V and VC. A V rhyme of course is $100 \%$ of itself in all positions. The average of VC rhymes word-finally, while notably above the average for word-final V, clearly falls short of the level of non-final unstressed VC rhymes. This of course is consistent with the weight behavior of these rhyme shapes: While VC is heavy in non-final positions it is light word-finally. Thus we can see the weight criterion as a proportional increase threshold that final VC falls short of.

Turning to the interval data, the percentages are shown calculated both with respect to the shortest weight domain in each position (VC non-finally and V finally) (in (10a)) and with respect to $\mathrm{VC}$ in all positions (in (10b)). 
a. VC baseline non-finally, $\mathrm{V}$ baseline _\#

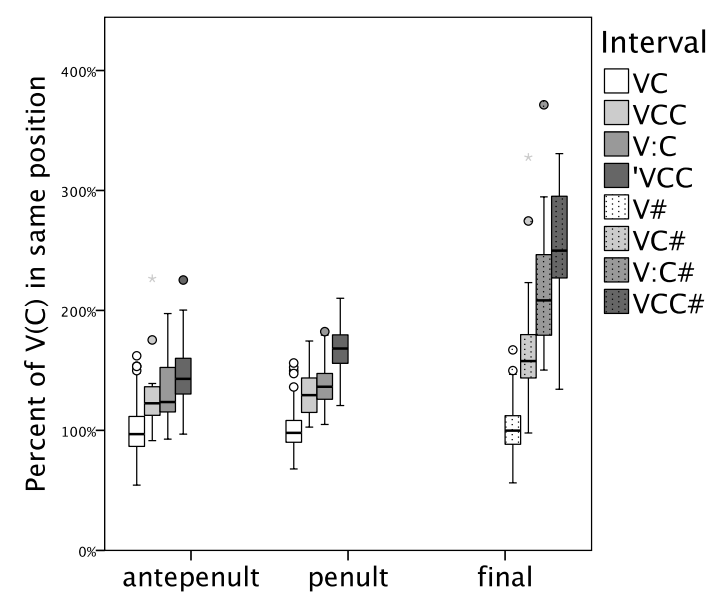

b. VC baseline in all positions

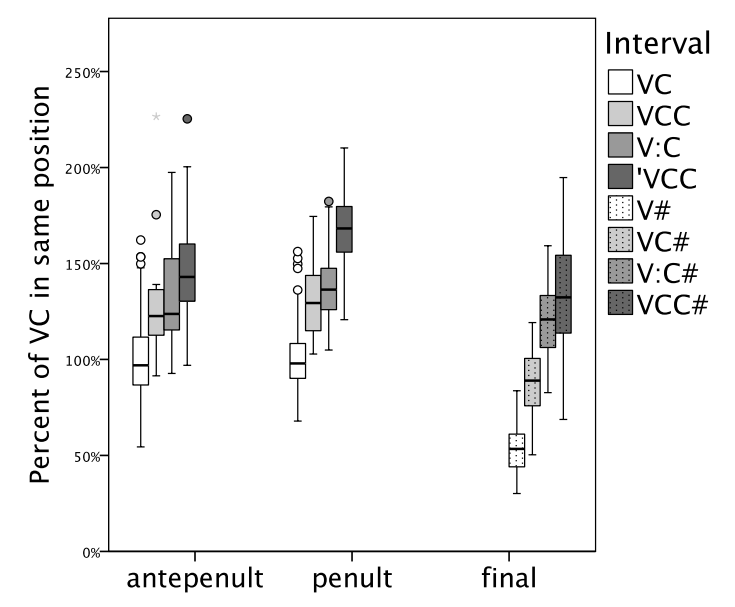

Looking at (10a) we can see that taking the shortest weight domain as the baseline in all positions, as was done for the rhyme data, does not result in a consistent picture for the interval durations, as a final VC interval is large and does not pattern with the final V interval. On the other hand, taking $\mathrm{VC}$ intervals as the baseline in all positions shows a more coherent picture (in (10b)). Now it is all VC intervals that are at the same level, since they are the average to which the others are compared. We do not find the extreme increases in word-final intervals that we see in (10a). Note that the non-final increase percentages are the same between the two graphs, but now the word-final percentages, like the rhyme data percentages, show a final VC falling notably short of the average proportional increase of non-final VC intervals. However, we see more overlap in non-final position of the light baseline weight domains and the heavy weight domains than we saw with the rhyme data in (7). This is shown and discussed in the following section.

3. Conclusion. Knowing that human perception works logarithmically, we examined the interval durations with the same approach as was used for rhyme durations in Lunden (2013). The rhyme durations from a set of Norwegian nonce words were known to fall into a pattern where heavy rhymes, on average, met a proportional increase threshold over the shortest light rhymes in the same position of the word, whereas light rhymes were known to fall short of this threshold on average. We found that if we take the $\mathrm{VC}$ interval as the baseline from which the percentages are calculated, as VC intervals are light in all positions, then we can find a similar pattern in the interval data. The rhyme and interval percentages are shown in (11) with a bar showing what might be identified as the proportional increase threshold. 
(11) Percentages with proportional increase thresholds

a. Rhymes: Percentage of same-position V

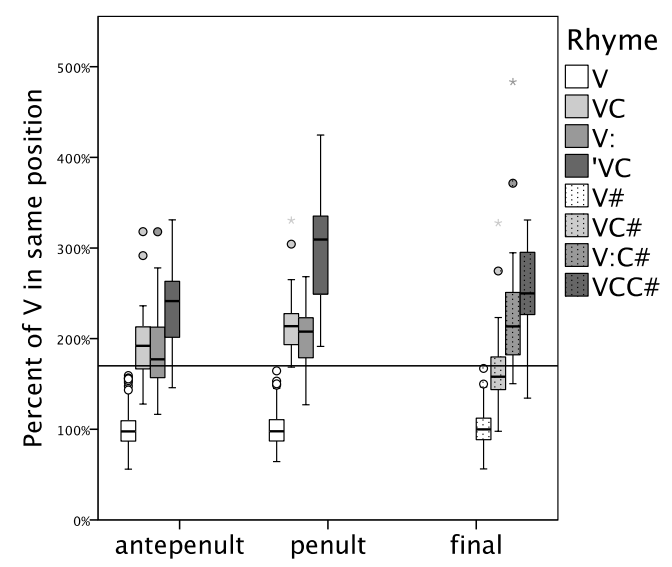

b. Intervals: Percentage of same-position VC

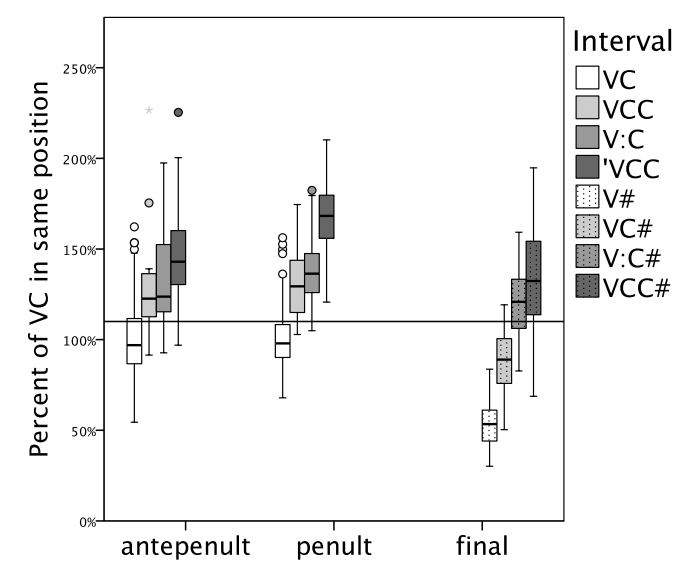

A threshold can be identified in both the rhyme percentages (in (11a)) and the interval percentages (in (11b)). However, the range non-final light intervals overlaps with some non-final heavy intervals much more than we see with rhyme percentages. While the threshold bar is at $170 \%$ in the rhyme graph, it is at $110 \%$ in the interval graph. Coupled with the heavy overlap between VC (light) and VCC (heavy) intervals, it appears that the interval data does not allow for as robust a proportional increase analysis of weight.

Reanalyzing the data reported on in Lunden (2013) with interval theory, I have shown that intervals exhibit some of the same duration-based relations to weight as was previously established for rhymes. It is clear that for any coherent proportional increase account, VC must be taken as the baseline interval for comparison in all positions of the word. The proportional increase of intervals over the same-position baseline interval was clear in the perception study data, where the interval model performed as well as the rhyme model. While the proportional increase account of intervals using the production study data was tenable, the overlap of non-final light intervals with some non-final heavy intervals, as well as a low proportional increase threshold raises questions about the perceptual clarity of the relations between intervals.

\section{References}

Broselow, Ellen, Susan Chen \& Marie Huffman. 1997. Syllable weight: Convergence of phonology and phonetics. Phonology 14. 47-82.

Garcia, Guilherme. 2017. Weight gradience and stress in Portuguese. Phonology 34(1). 41-79. http://dx.doi.org/10.1017/S0952675717000033.

Goedemans, R.W.N. 1998. Weightless segments: A phonetic and phonological study concerning the metrical irrelevance of syllable onsets LOT Dissertations No 9. The Hague: Holland Academic Graphics.

Gordon, Matthew. 2002. A phonetically-driven account of syllable weight. Language 78. 51-80. 
Hirsch, Aron. 2014. What is the domain for weight computation: the syllable or the interval? In Proceedings of the Annual Meetings on Phonology, vol. 1 , http://dx.doi.org/10.3765/amp.v1i1.21.

Lehiste, Ilse. 1970. Suprasegmentals. Cambridge, MA: MIT Press.

Lunden, Anya. 2011. The weight of final syllables in English. In Proceedings of the 28th West Coast Conference on Formal Linguistics, 152-159. http://www.lingref.com/cpp/wccfl/28/index.html.

Lunden, Anya. 2013. Reanalyzing final consonant extrametricality: A proportional theory of weight. Journal of Comparative Germanic Linguistics 16(1). 1-31. http://dx.doi.org/10.1007/s10828-013-9053-3.

McCarthy, John. 1979. Formal problems in semitic phonology and morphology. Cambridge, MA: Massachusetts Institute of Technology dissertation.

Oller, D.K. 1973. The effect of position in utterance on speech segment duration in English. Journal of the Acoustical Society of America 54(5). 1235-1247. http://dx.doi.org/10.1121/1.1914393.

Prince, Alan \& Paul Smolensky. 1993. Optimality theory: Constraint interaction in generative grammar. Ms., Rutgers University, New Brunswick and University of Colorado, Boulder.

Ryan, Kevin. 2014. Onsets contribute to syllable weight: Statistical evidence from stress and meter. Language 90(2). 309-341. http://dx.doi.org/10.1353/lan.2014.0029.

Steriade, Donca. 2012. Intervals vs. syllables as units of linguistic rhythm. Handouts, EALING, Paris. 